\title{
What should we expect in a prognosis study in 2012?
}

\author{
Rory Hachamovitch, MD, MSc
}

\section{See related article, pp. 907-913}

Among the leading justifications for the use of stress radionuclide myocardial perfusion imaging (MPI) is the wealth of published literature demonstrating the incremental prognostic value of this test over other available data. ${ }^{1}$ These reports examine various cohorts (all-comers, diagnostic patients, known CAD, etc.), multiple clinical scenarios (post-MI, preoperative cardiovascular clearance, assessment of suspected CAD, etc.), different types of stress, types of tracers, etc. Although many believe that most of what needs to be known with respect to the prognostic implications of MPI has already been reported, and that there is very little remaining to be done, this is far from the case. The gaps in our knowledge base that are most striking include

1. Whether the results reported to date with shorter follow-up (most published studies are limited to 1-2 years) will also be the case with longer follow-up (e.g., >5-10 years).

2. What the prognostic impact of post-MPI treatment is on patient outcome, and whether basing post-test patient management on MPI results enhance patient outcomes.

3. How does patient selection optimize the MPIoutcome association, especially whether the application of appropriateness criteria enhance MPI-related risk stratification?

Other knowledge gaps exist and similar questions can be posed.

\footnotetext{
From the Cardiovascular Imaging Section, Department of Cardiovascular Medicine, Heart and Vascular Institute, Cleveland Clinic, Cleveland, $\mathrm{OH}$.

Reprint requests: Rory Hachamovitch, MD, MSc, Cardiovascular Imaging Section, Department of Cardiovascular Medicine, Heart and Vascular Institute, Cleveland Clinic, Cardiovascular Medicine J1-5, Euclid Avenue, Cleveland, OH 44195; hach@msn.com.

J Nucl Cardiol 2012;19:883-6.

1071-3581/\$34.00

Copyright (C) 2012 American Society of Nuclear Cardiology.

doi:10.1007/s 12350-012-9611-0
}

Not only is the extent of this prognostic literature noteworthy but also it is important to note that the quality of these studies has dramatically improved. ${ }^{1-3}$ The statistical tools that are applied are often more rigorous, the power of the studies is far greater than in the past, and the questions posed are more sophisticated. ${ }^{2,3}$ Despite this, although we can claim a large prognostic literature, it is a methodologically problematic literature. There are almost no prospective randomized clinical trial results that can directly address whether and when MPI should be used and how to change patient management based on the results. Thus, practically, we are dependent on observational studies - usually single site - to support the use of MPI. The implications of this limitation include the increased level of analytic complexity necessary with these types of studies, including their design, analysis, interpretation, and understanding by journal readership. ${ }^{4-6}$

Thus, in light of the extensive published prognostic literature and the need to perform the complex, associated analyses in a sound manner, the question must be asked "what are reasonable expectations to have from a study examining the prognostic value of stress myocardial perfusion SPECT in 2012?', The study by Boiten et $\mathrm{al}^{7}$ in the current issue of this journal exemplifies a number of these expectations. These authors sought to evaluate the long-term prognostic value of exercise stress Tc99m tetrofosmin SPECT for the prediction of adverse cardiac events in patients with known or suspected coronary artery disease presenting to testing in their institution.

The current study by Boiten and colleagues examines the long-term prognostic value of bicycle stress SPECT in a cohort of 655 patients who underwent testing and were followed for a mean of 11.0 years. Their prognostic analysis is based on 638 patients among whom 174 all-cause deaths, 76 non-fatal myocardial infarctions, and 194 late revascularization procedures ( $>60$-day post-MPI) occurred. They report "Univariable and multivariable Cox proportional hazard regression analysis showed that exercise $99 \mathrm{mTc}-$ tetrofosmin SPECT provided prognostic information incremental to clinical data and exercise test data," ${ }^{7}$ and conclude that "...myocardial perfusion SPECT has an incremental long-term prognostic value over clinical and stress test parameters for the prediction of major adverse cardiac events.", 7 As is always the case, the reader must 
ask whether the internal validity and external validity of the paper falls within the range of acceptability. ${ }^{2,4} \mathrm{~A}$ crucial question for the reader to ask is on what basis the authors reached their stated conclusion.

To date, there are several specific aspects of prognostic analyses that must be kept in mind when this type of study is read. First, for a reader to understand both the results and how to apply them in practice, the MPI metrics must be presented in a format that is easy for a non-nuclear cardiologist to interpret. Although presenting the MPI results as a "normal" versus "abnormal" result is the simplest approach, it also has been shown to minimize the potential information provided by the test. While summed scores are often presented (based on the generally accepted 5-point, 17-segment model), "translation" of summed scores into a \%myocardium may enhance the generalizability of the results. ${ }^{8}$ In this study, these metrics are presented as summed scores based on a 47-segment, 4-point scoring system. Hence, even for experienced nuclear cardiologists, the results of the current study are potentially misleading and difficult to interpret.

Study power (number of events occurred) will often drive how the analysis can and will be done. ${ }^{2,5}$ If many deaths occurred during follow-up, the study can be approached as a true survival study. If not, the authors may need to examine potential composite endpoints. Although their use improves study power, composite endpoints assume risk homogeneity among the component events, thus, results may materially change with the addition of endpoints and conclusions must be examined more carefully. ${ }^{2}$ In the setting of randomized clinical trials, physician-driven outcomes (use of catheterization, revascularization, hospitalization, etc.) are often more amenable to the intervention examined compared to hard events, and more frequently have positive results. ${ }^{2}$ In this study, the authors report that their primary endpoint was a composite of three events (all-cause death, non-fatal myocardial infarction, and late revascularization). Although we are told how many of each occurred, we do not know how many total patients had the primary endpoint. Nonetheless, this study is very well powered. Indeed, the question of why the authors would choose to include "softer" endpoints such as late revascularization as part of a primary endpoint when sufficient power could be achieved using all-cause death alone is unclear. As revascularization procedures and MI tend to be associated with different characteristics than all-cause death, this is an important consideration.

One of the most interesting issues in prognostic analyses is how to handle patients referred to early revascularization after MPI. ${ }^{2}$ Historically, these patients have been censored from analyses on grounds that this early intervention altered outcomes and, as it was based on the MPI results, would introduce a selection bias. ${ }^{9,10}$ More recently, the argument has been made that the bias introduced by censoring is greater than that present if these patients are left in the study., ${ }^{2,11,12}$ If these patients are left in the study they can be adjusted for or used as a stratification factor in the modeling process, thus, the bias can be adjusted for, to some extent, in the analysis. How early revascularizations should be defined (those occurring in the first 60,90 , or 120 days) and whether or not early revascularizations should be differentiated from all revascularizations in this setting is unclear.

Examination of early revascularization rates is also important in another context. The magnitude of bias introduced by post-MPI referral to revascularization is a function of the pattern of post-MPI resource utilization at the particular institution where the data was collected. $^{2}$ The proportion of patients with extensive ischemia referred to revascularization after MPI varies among centers, physicians, and by patients' cardiac history, symptoms, stress test results, etc. ${ }^{8,13,14}$ Probably the most unique result of this study is that of $>600$ patients reported, only 10 patients were referred to postMPI revascularization. This represents $<3 \%$ of patients with abnormal studies and $<6.5 \%$ of patients with ischemia by MPI. This result represents a remarkably low rate of revascularization that has serious implications. First, from a health outcomes perspective, this finding raises questions regarding how referring physicians are using this modality. The authors put forward the explanation that in patients with ischemia, “...medical therapy was optimized before a decision on referral to percutaneous coronary revascularization or surgical coronary revascularization was made," but no such data is shown. Results from a multicenter registry recently published suggest that this is not the case, and that medical therapy is poorly addressed after MPI. ${ }^{13} \mathrm{An}$ obvious question is how to justify the use of MPI, not an inexpensive modality, when the results do not alter patient management. Furthermore, if a trial of aggressive medical therapy is to be the initial strategy, why are the test results necessary?

The second implication of these revascularization rates is that compared to other cohorts in other studies from other institutions, due to this exceedingly low revascularization rate, this cohort is arguably "debiased" with respective to the biases created by selective post-test referral to revascularization. ${ }^{2}$

Finally, what a reader should expect in a results section is also important. To determine whether the results of the study are generalizable to the patients seen by the reader, a careful review of the characteristics and demographics of the study cohort is necessary. This fundamental step cannot be taken with the current cohort, as this study is results-poor, the results section 
limited to three short paragraphs. We are told remarkably little about these patients with respect to a characterization of whom they are and many basic descriptors (e.g., prior revascularization, results of stress testing, multiple clinical characteristics) are not presented.

In addition to a demographic characterization of the cohort, studies often present unadjusted event rates or hazard ratios for individual predictors. This practice is problematic. On the one hand, many readers may be reassured by seeing that specific patient characteristics are associated with greater risk (e.g., diabetes mellitus, renal function, age, etc.). Alternatively, these results are problematic due to the bias and confounding associated with this study design-is the univariable result "real" or is it because some other factor was not adjusted for $?^{2,5,15}$ For example, in this study, diabetes mellitus and smoking are only significant predictors of adverse events after they are considered with other variables, but are not significant predictors of outcomes when considered alone. Conversely, several stress test variables and hypercholesterolemia are significant initially as individual predictors, but are no longer significant after risk adjustment. Thus, whether presenting unadjusted associations of individual variables with the primary endpoint is helpful or confusing must be considered.

Finally, and of greatest importance, given that this is a non-randomized, observational study, any final conclusions must be based on and supported by the results of multivariable modeling. In this study, little is told to us of how the models were developed. Whether model assumptions are examined is not mentioned. Covariate selection is a very important consideration, and a study's methods section must indicate whether the pre-imaging data that was adjusted for was pre-specified (pre-defining which variables will be entered into the model on the basis of previously published studies or clinical experience) or whether an alternative approach was used., 2,5,15 Covariate pre-specification is advantageous in that it enhances the statement of the hypothesis and eliminates the potential need for statistical correction for multiple testing of the data (adjustment of $\alpha$ ). Also, the use of stepwise models, forward or backward, have a greater than appreciated error rate in identifying key predictors. ${ }^{5,16}$

In this study, the modeling results are presented in a minimalist manner. Based on the results presented in Table 2, it appears that the authors examined multiple, overlapping nuclear variables. How this issue was handled in the modeling process is not mentioned. The message put forward is that the strength of association between a summed stress score and adverse outcomes is significant after adjusting for patient age, diabetes mellitus, smoking, and typical angina during exercise, assuming that the model was properly developed and assumptions examined. Despite having a very robust dataset (many events), the authors "underfit", their model, putting in considerably fewer covariates than permissible, omitting several that would be considered important. The modeling results are also problematic to interpret in other respects. In the unadjusted analysis, per unit increase in summed stress scores predicted about a two and a half times increase in patient risk [hazard ratio $2.55(1.82,3.57)]$. Per unit increase in summed difference score and summed rest score increased risk 29 and $8 \%$, respectively [hazard ratios 1.29 (1.20-1.39) and 1.08 (1.01-1.12)]. Thus, the clinical value and/or implications of the model presented are uncertain; the generalizability to the practice of the average clinician is unclear.

As can be appreciated from the above, the analytic approach to be taken in a prognosis study must be carefully planned and specific issues addressed. It is important to note that many of these issues do not have a "wrong" or "right" decision, but require a series of well thought out decisions by the authors with explanations of why specific approaches were taken, and the advantages and disadvantages of the alternatives. These steps are not necessary for their own sake. A rigorous approach to statistical modeling usually yields more accurate and meaningful results, and may yield key clinical insights. This is important not only in the context of scientific method and methodical accuracy but also to improve internal and external validity of the results. These studies must pass muster not only with the imaging community but also with non-imaging physicians and, increasingly, payers. The future utilization of our modality lay in the balance.

\section{References}

1. Shaw LJ, Hage FG, Berman DS, Hachamovitch R, Iskandrian A. Prognosis in the era of comparative effectiveness research: Where is nuclear cardiology now and where should it be? J Nucl Cardiol 2012 (Epub ahead of print).

2. Hachamovitch R, Di Carli MF. Contemporary reviews in cardiovascular medicine: Methods and limitations of assessing new noninvasive tests II. Outcomes-based validation and reliability assessment of noninvasive testing. Circulation 2008;117:2793801.

3. Hachamovitch R, Shaw L, Berman DS. Methodological considerations in the assessment of noninvasive testing using outcomes research: pitfalls and limitations. Prog Cardiovasc Dis 2000; 43:215-30.

4. Hachamovitch R, Di Carli MF. Contemporary reviews in cardiovascular medicine: Methods and limitations of assessing new noninvasive tests: I. Anatomy-based validation of noninvasive testing. Circulation 2008;117:2684-90.

5. Harrell FE Jr, Lee KL, Mark DB. Multivariable prognostic models: issues in developing models, evaluating assumptions and 
adequacy, and measuring and reducing errors. Stat Med 1996;15: 361-87.

6. Harrell FE Jr, Lee KL, Matchar DB, Reichert TA. Regression models for prognostic prediction: advantages, problems, and suggested solutions. Cancer Treat Rep 1985;69:1071-7.

7. Boiten $\mathrm{H}$, van der Sijde J, Ruitinga $\mathrm{P}$, et al. Long-term prognostic value of exercise technetium-99m tetrofosmin myocardial perfusion single-photon emission computed tomography. J Nucl Cardiol 2012. doi:10.1007/s12350-012-9585-y.

8. Hachamovitch R, Hayes SW, Friedman JD, Cohen I, Berman DS. Comparison of the short-term survival benefit associated with revascularization compared with medical therapy in patients with no prior coronary artery disease undergoing stress myocardial perfusion single photon emission computed tomography. Circulation 2003;107:2900-7.

9. Pryor DB, Harrell FE Jr, Lee KL, et al. Prognostic indicators from radionuclide angiography in medically treated patients with coronary artery disease. Am J Cardiol 1984;53:18-22.

10. Staniloff HM, Forrester JS, Berman DS, Swan HJ. Prediction of death, myocardial infarction, and worsening chest pain using thallium scintigraphy and exercise electrocardiography. J Nucl Med 1986;27:1842-8.

11. Hachamovitch R, Berman DS, Kiat H, Cohen I, Friedman JD, Shaw LJ. Value of stress myocardial perfusion single photon emission computed tomography in patients with normal resting electrocardiograms: An evaluation of incremental prognostic value and cost-effectiveness. Circulation 2002;105:823-9.

12. Hachamovitch R, Rozanski AR, Hayes SW, et al. Predicting therapeutic benefit from myocardial revascularization procedures: are measurements of both resting left ventricular ejection fraction and stress-induced myocardial ischemia necessary? J Nucl Cardiol 2006;13:768-78.

13. Hachamovitch R, Nutter B, Hlatky MA, et al. Patient management after noninvasive cardiac imaging results from SPARC (Study of myocardial perfusion and coronary anatomy imaging roles in coronary artery disease). J Am Coll Cardiol 2012;59:462-74.

14. Hachamovitch R, Hayes SW, Friedman JD, et al. Is there a referral bias against revascularization of patients with reduced $L V$ ejection fraction? Influence of ejection fraction and inducible ischemia on post-SPECT management of patients without history of CAD. J Am Coll Cardiol 2003;42:1286-94.

15. Greenland S. Modeling and variable selection in epidemiologic analysis. Am J Public Health 1989;79:340-9.

16. Harrell FE Jr. Resampling, validating, describing and simplifying the model. Regression modeling strategies. New York: Springer; 2001. p. $87-103$. 